\title{
Variedades de Pomáceas (Chile y Cuyo 1700-1850)
}

\author{
Pome fruit varieties (Chile and Cuyo 1700-1850) \\ Pablo Lacoste ${ }^{1}$; José Antonio Yuri ${ }^{2}$; Marcela Aranda ${ }^{3}$; Amalia Castro ${ }^{4}$; Katherine Quinteros; \\ Mario Solar; Natalia Soto; Cristian Chávez
}

\section{RESUMEN}

El estudio hace visibles las variedades de Pomáceas (manzanos, perales y membrillos), cultivadas en Chile y Cuyo entre 1700 y 1850. El trabajo se funda en el corpus documental de fondos notariales y judiciales de los archivos de Chile, San Juan y Mendoza. Se contabilizaron más de 1.000 plantas, representadas por once variedades en el caso de los perales, siete de manzanos y sólo dos de membrillos. Fue posible detectar cierta continuidad del cultivo de frutales en Chile con el observado en la fruticultura musulmana de al-Andalus en España.

Palabras clave: Agricultura tradicional, fruticultura, Pomáceas, variedades.

\begin{abstract}
The present study illustrates the traditional Pome (apple, pear and quince) fruit varieties grown in Chile and Cuyo between 1700 and 1850. The data of the study were obtained from notary and justice archives of Chile, San Juan and Mendoza. Eleven varieties of pear, seven of apple and two varieties of quince were identified. Also, some correspondence with the Muslim fruit culture of al-Andalus in Spain was detected.
\end{abstract}

Key words: Traditional agriculture, fruit culture, Pome fruit, fruit variety.

Los estudios de secuencia de ADN han permitido ampliar el conocimiento sobre la identidad y el origen de las plantas, como así también las relaciones de unas con otras. A su vez, para una interpretación más rica de estos resultados, resulta conveniente complementarlo con los aportes de documentos históricos (Ibáñez et al., 2011). Para avanzar en esta dirección, los investigadores del campo de la agronomía han comenzado a revalorizar las antiguas fuentes, particularmente las ampelográficas y tratados de agricultura (Cabello Sáenz, 2011). De todos modos, la información más rica sobre el tema se encuentra en otros documentos, en especial en los inventarios de bienes levantados durante siglos por los notarios con fines judiciales. Esta fuente está normalmente fuera del ámbito de los agrónomos, pero es familiar para los historiadores. El complemento de ambos enfoques para comprender mejor la evolución e identidad de una variedad puede verse en el caso de la vid torrontés, examinado tanto desde los marcadores de ADN (Agüero et al., 2003), como desde los documentos históricos (Lacoste et al., 2010). El presente artículo procura extender dicho estudio a las variedades de Pomáceas (manzanos, perales y membrillos) cultivadas en Chile y Cuyo entre 1700 y 1850.

1 Profesor Titular de la Universidad de Santiago de Chile (USACH). Instituto IDEA. Román Díaz 89, Providencia, Santiago de Chile. Teléfono (56-2) 7181360. Correo electrónico: pablo.lacoste@usach.cl

2 José Antonio Yuri, director del Centro de Pomáceas de la Universidad de Talca. 2 Norte 685. Talca, Chile. Teléfono (56-71) 200200. Correo electrónico: ayuri@utalca.cl

3 Marcela Aranda es doctoranda USACH. Instituto IDEA. Román Díaz 89, Providencia. Santiago de Chile. Teléfono (56-2) 7181360 Correo electrónico: marcela.aranda06@gmail.com

4 Amalia Castro es profesora de la Universidad Católica Silva Henríquez. General Jofré 462. Santiago de Chile. Chile. Teléfono (56-2) 4601100. Correo electrónico: castrosancarlos@yahoo.com.mx 
Se han estudiado las jurisdicciones de $\mathrm{La}$ Serena, San Felipe, Santiago, San Fernando, Parral, Cauquenes, Mendoza y San Juan, mediante la compulsa de documentos originales inéditos, principalmente inventarios de bienes, obrantes en los fondos del Archivo Nacional de Santiago (AN), el Archivo Histórico de Mendoza (AHM), el Archivo General de la Provincia de San Juan (AGPSJ) y el Archivo del Poder Judicial de San Juan (APJSJ). El corpus documental ha entregado información sobre la variedad de 1.032 ejemplares de Pomáceas (764 perales, 254 manzanos y 14 membrillos). Se distinguen las variedades cultivadas, atendiendo cuáles se registraron, dónde, cuándo y cuál fue su valor económico, entre otros datos.

El estudio de las variedades antiguas del Reino de Chile tiene que colocarse en su contexto histórico. Para ello, lo más conveniente es compulsarlo con los manuales de los agrónomos y botánicos musulmanes de al-Andalus, que también se interesaron por identificar y clasificar las variedades de frutales. En efecto, a fines de la Edad Media los manuales andalusíes habían detectado distintas variedades de estas frutas. Por los criterios utilizados, esa clasificación resulta de utilidad para interpretar con mayor claridad el presente trabajo.

Dentro del manzano, los manuales andalusíes destacaban el sabi que no florece y está desprovisto de semillas (azurronado o vulgar); el alibi (pintado o señalado); el rujami (marmóreo), debido a su tersa blancura; el sabarqn; el bermejo y otros (Carabaza Bravo et al., 2004). También se identificaron variedades de membrillo, sobre todo "cultivado y silvestre, éste de tamaño muy pequeño; de fruto redondo, que puede ser pequeño o grande y de fruto alargado, llamado minhad, del que hay una especie dulce y otra agria" (2004: 131). Con respecto al peral destacaron "hay montesinos y hortense; azucarado y agrio, acalabazado, acandilado y otros; hay peral dulce, agrio, poco jugoso, de mucho jugo, grande, mediano y pequeño" (2004: 141).

Esta temprana clasificación de variedades se fundaba en aspectos como el color, la forma, el tamaño, el sabor, y el lugar de cultivo (monte o huerto). De esta manera se comenzó a transitar el camino de la identificación de las variedades de Pomáceas en la península ibérica, cultura que posteriormente se trasladó a América.

Las luchas de poder entre musulmanes y cristianos dentro de la península ibérica determinaron una ruptura en los canales de comunicación de los conocimientos entre ambas comunidades. Como resultado, los avances de los agrónomos andalusíes no fueron debidamente recogidos ni difundidos por los españoles, al menos en forma oficial. Los tratados de los agrónomos españoles, escritos después, no fueron capaces de continuar el proceso de construcción de conocimiento generado por sus antecesores españoles. Las obras de Alonso de Herrera (1513) y Miguel Agustín (1617) no entregaron detalles sobre variedades de Pomáceas. La única referencia al tema es muy vaga y se limita a mencionar la existencia de numerosas variedades de peral: "de pocos o de ningunos frutales hay tantas especies como del peral, por cuanto le es muy natural el injerir y mezclarse con cualquier otro árbol, y así hay peras de verano, otoño invierno y todo el año" (Agustín, 1617). Estas definiciones parecen pobres, al compararse con el mayor grado de definición alcanzado por los agrónomos andalusíes unos siglos antes.

La ausencia de mayores precisiones sobre variedades de frutas en general y Pomáceas en particular en los tratados de Alonso de Herrera y Fray Agustín muestra la brecha que, en lo referente al interés por los frutales, existió entre los musulmanes y los cristianos en la Europa medieval. En los pueblos cristianos, los alimentos de origen vegetal eran cereales, legumbres y vino. Consistente con el interés de su entorno social, el manual de Alonso de Herrera está orientado en esta dirección: el libro primero de su obra lo dedica a cereales y legumbres, y el libro segundo a la vid y el vino. Para esas plantas el autor sí entrega datos sobre "castas" o variedades, a través de un aporte de singular valor, pues "realiza las primeras descripciones que nos permiten identificar variedades actuales" (Cabello Sáenz, 2011). Sin embargo, en esa obra no se incluyen otros frutales.

Esta situación contrasta notablemente con la cultura musulmana, la cual sí se interesaba vivamente por los frutales y la fruta. Ésta no era un alimento reservado a las élites, sino de amplio consumo popular, tal como se reflejaba en los zocos. Resulta por tanto natural que los agrónomos y botánicos andalusíes se hayan interesado por los frutales y los hayan incluido en sus tratados.

A pesar del desencuentro político entre moros y cristianos, la tradición musulmana de apreciación de los frutales se transmitió al pueblo español mediante el roce sociocultural. 
POMÁCEAS EN CHILE

Distribución Geográfica de Plantas

La Serena, San Felipe, Santiago, San Fernando, Parral y Cauquenes (1700 - 1860)

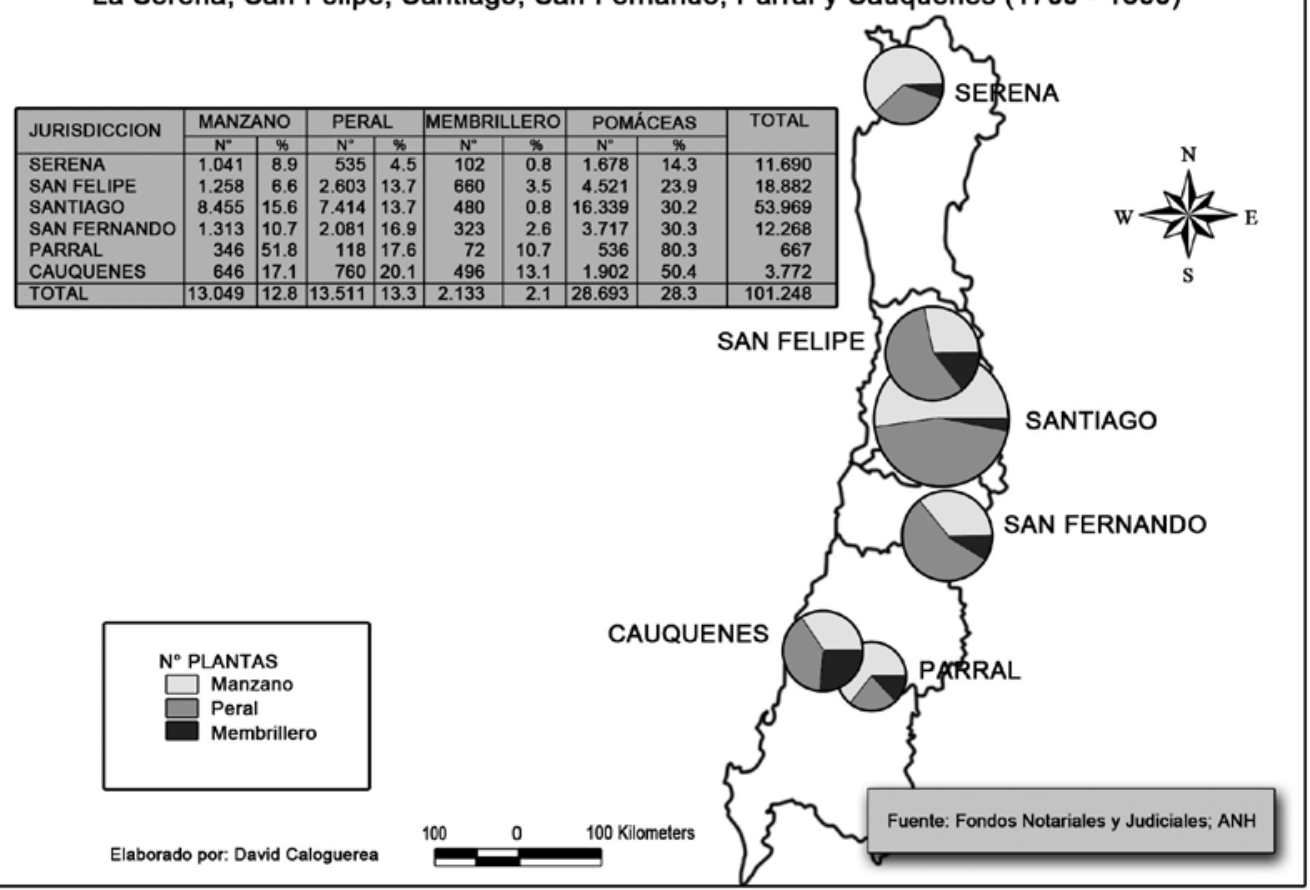

POMÁCEAS EN CUYO

Distribución Geográfica de Plantas

San Juan y Mendoza (1700-1870)

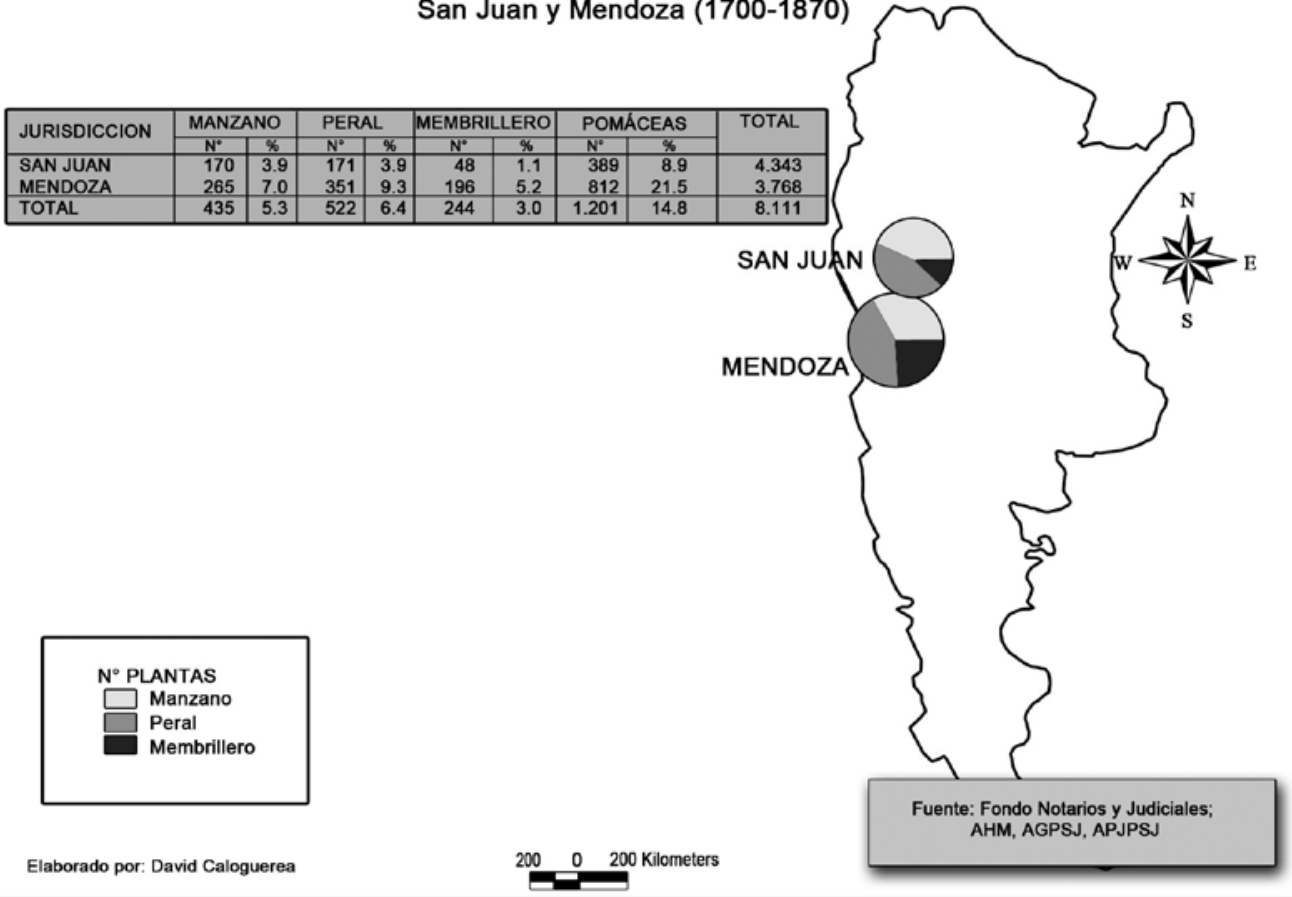

Figuras 1 y 2. Distribución geográfica de Pomáceas en Chile entre 1700 y 1860, expresada en número de plantas y porcentaje. 
Los conquistadores españoles, que a partir del siglo XVI comenzaron la colonización del Reino de Chile, llevaron consigo la cultura de la apreciación de la fruta, heredada de los pueblos musulmanes. Poco a poco, a partir del siglo XVIII, comenzaron a observarse e identificarse las variedades de carozos en Chile, tal como quedó documentado en los inventarios de bienes, testamentos y demás expedientes judiciales (Cuadros 1, 2 y 3), uno para cada especie de Pomáceas.

De acuerdo al Cuadro 1 los fruticultores de Chile y Cuyo cultivaron once variedades de perales, siete de manzanos y dos de membrillos. Siguiendo criterios parecidos a los de la botánica musulmana, las variedades de Pomáceas del Reno de Chile se nucleaban en torno a grupos:

a) origen geográfico: peral chino, armenio, joaquino y portugués; manzano quillotano y joaquino ${ }^{1}$; b) ciclo de maduración y cosecha: peral cuaresmero, peral invernizo; c) forma o tamaño de la fruta: peral redondo, peral a libra; d) tiempo de duración para el consumo: peral de guarda; manzano de guarda; e) gusto: manzano dulce y ácido; membrillo dulce; f) parecido de su fruta con la de otra planta: peral bergamoto, peral cerezo; manzano pero; membrillo lúcumo; g) otro: manzano camueso.

La presencia de estas plantas en Chile y Cuyo no fue uniforme. Existen singulares diferencias de tiempo, espacio y frecuencia. Desde el punto de vista cronológico, las variedades pueden distinguirse entre tempranas (comenzaron a detectarse en la primera mitad del siglo XVIII), medias (fines de esa centuria) y tardías (recién aparecieron en el siglo XIX). También se pueden clasificar de acuerdo a la frecuencia: había variedades con alta, media, baja o muy baja frecuencia. Los cuadros entregan detallada información al respecto, señalando el lugar, año y cantidad de ejemplares detectados para cada variedad. Es importante señalar un dato que no se ha volcado en ellos: en la estancia Cucha Cucha, donada a Ramón Freire como premio por los servicios prestados durante la guerra de la Independencia, había un manzanar con 60 manzanos peros, los cuales, además, eran reconocidos como de guarda.

Los precios de mercado también muestran distintas valoraciones para las diferentes variedades de peral. El inventario de bienes de don Bernardo Ramírez (Guacaragua, partido de Colchagua, 1811), resulta esclarecedor por entregar información simultánea para muchas de ellas. Los tasadores consideraron que los más barato eran el Peral redondo ( $3 \frac{1}{2}$ reales), el Peral cerezo y el Peral cuaresmero (4 reales por planta) ${ }^{2}$. En un nivel intermedio estaba el Peral a libra (6 reales). El mayor valor correspondía al Peral bergamoto: 5 reales el pequeño y 8 reales el grande, lo mismo que el Peral armenio. Por su parte, los tres ejemplares de Peral cerezo, que tal vez por estar injertados daban peras bergamotas, se tasaban a 6 reales ${ }^{3}$.

\section{Conclusión}

Durante el período colonial y hasta mediados del siglo XIX, la fruticultura chilena alcanzó una interesante diversidad de especies de Pomáceas. Se llegaron a identificar once variedades de perales, siete de manzanos y dos de membrillos. Por el criterio utilizado para construir la clasificación y las denominaciones de esas variedades, es posible interpretar que hubo un lazo entre la fruticultura musulmana de al-Andalus y el Reino de Chile.

La cultura de apreciación de los frutales y la fruta, introducida en la península ibérica por los musulmanes, estudiada y sistematizada por los agrónomos y botánicos andalusíes, tuvo cierta influencia en el Reino de Chile. Esta continuidad llama la atención porque los reyes y líderes españoles, tras derrotar militarmente a los musulmanes, menospreciaron buena parte de sus aportes, incluyendo sus avances científicos en el campo de la agronomía y la botánica. En el plano específico de los estudios de variedades de Pomáceas, los avances logrados por los tratados andalusíes fueron ignorados por los textos agrícolas españoles de los siglos XVI y XVII. Éstos priorizaron otras plantas, sobre todo la vid y los cereales, tal como se había estilado en la cultura medieval europea, en la cual hubo poco interés por la fruticultura.

No obstante, a pesar del desencuentro entre los gobernantes, la cultura de la apreciación de la fruta se transmitió mediante el roce sociocultural y se propagó hacia la España cristiana. Posteriormente, los conquistadores españoles que a partir del siglo XVI llegaron a Chile introdujeron y propagaron la antigua tradición árabe de apreciación de los frutales, con la natural adaptación a la realidad americana. A partir del siglo XVIII comenzó a hacerse visible la rica diversidad de variedades de frutales del Reino de Chile.

Las plantas frutales nativas o las nuevas variedades aparecidas en el Reino de Chile carecen 
Cuadro 1. Variedades de perales en Chile y Cuyo (1726-1848)

\begin{tabular}{|c|c|c|c|c|c|c|c|c|c|c|c|}
\hline Localidad & Año & Fruticultor & BER & CER & RED & ARM & POR & $\mathrm{CU}$ & $\mathrm{CHI}$ & Otro & Total \\
\hline San Felipe & 1726 & Joana Margarita & 2 & & & & & & & & 2 \\
\hline Santiago & 1745 & Francisco Laguna & 3 & 3 & & & & & & & 6 \\
\hline Hacienda Elqui - La Serena & 1748 & Magdalena González & 5 & & & & & & & & 5 \\
\hline La Serena & 1758 & Francisco Godoy & 4 & & 4 & & & & & & 8 \\
\hline Copiapó & 1767 & Compañía de Jesús & 4 & & & & & & & & 4 \\
\hline Estancia Comiella - S. Fndo. & 1774 & José Quesada & 11 & 10 & 13 & & & & & & 34 \\
\hline La Serena & 1785 & Antonia Bravo & 1 & & & & & & & & 1 \\
\hline Rapel -San Fernando & 1789 & Agustín de la Huerta & & 7 & & & & & & & 7 \\
\hline Loncomilla - Cauquenes & 1790 & Bernardo Amigo & & 1 & & $\mathrm{~s} / \mathrm{d}$ & & & 1 & & 2 \\
\hline Cauquenes & 1790 & Juana Salgado & 3 & & 2 & & & & & & 5 \\
\hline La Serena & 1791 & Bernarda Aguirre & 2 & & 1 & & & & & & 3 \\
\hline Curimón - San Felipe & 1791 & Jerónimo Ojeda & 7 & 14 & & 3 & & & 3 & 3 & 23 \\
\hline San Juan & 1792 & A M L de Guevara & 11 & & 17 & & & & & & 28 \\
\hline Estancia Auquinco - S. Fndo. & 1792 & Mateo Arenas & & 11 & 11 & & & & & & 22 \\
\hline San Fernando & 1793 & Manuela del Pino & & 3 & & & & & & & 3 \\
\hline Nancagua - San Fernando & 1793 & Rosa Paredes & & 7 & & & & & & & 7 \\
\hline Pelequén - San Fernando & 1794 & Pedro Cartagena & 2 & & 5 & & & & & & 7 \\
\hline Río Claro - San Fernando & 1795 & Mónica Morales & & & 5 & & & & & & 5 \\
\hline San Fernando & 1796 & José Riveros Aliaga & & 4 & 4 & & & & & & 4 \\
\hline San Fernando & 1797 & Mónica Román & & 5 & & & & & & & 5 \\
\hline Río Claro - San Fernando & 1798 & Nicolás Suasnabar & & 4 & & & & & & 1 & 5 \\
\hline San Felipe & 1803 & Juan José Olivares & & & 1 & & & & & & 1 \\
\hline Cauquenes & 1807 & Juana Salgado & 3 & & 2 & & & & & & 5 \\
\hline Los Andes & 1809 & Esteban Herrera & 22 & 14 & & & & & & & 36 \\
\hline San Juan & 1810 & Pedro Cortínez & 5 & & 1 & & & & & & 6 \\
\hline Guacaragua - San Fernando & 1811 & Bernardo Ramírez & 9 & 23 & 4 & 1 & & 7 & & 1 & 45 \\
\hline Tunca - San Fernando & 1812 & Juan Bautista Cabezas & 3 & 13 & 1 & & & & & & 17 \\
\hline Río Claro - San Fernando & 1812 & José Dieser & 3 & & & & & & & & 3 \\
\hline Villa San Fernando & 1812 & Manuel Gálvez & 1 & 6 & 1 & & & & & & 8 \\
\hline Manantiales - San Fernando & 1812 & Ma Tránsito Valenzuela & & 5 & 1 & & & & & & 6 \\
\hline Mendoza & 1813 & Josefa Antúnez & 1 & & & 1 & & & & & 2 \\
\hline Cauquenes & 1814 & Ma Angélica Durán & 2 & & & & & & & & 2 \\
\hline Maipú - Santiago & 1814 & Antonio Fernández & 1 & 4 & 4 & 3 & & & & & 12 \\
\hline Río Claro - San Fernando & 1814 & Feliciano Dieces & 1 & 9 & 5 & & & & & & 15 \\
\hline Valle Guacargüe - S. Fndo. & 1815 & Juan de Dios Horta & & 4 & & & & & & & 4 \\
\hline Traiguén - Cauquenes & 1816 & Ma. del Carmen Pereira & 4 & & & & & & & & 4 \\
\hline San Fernando & 1816 & Francisco Sosa & 3 & 1 & & & & & 1 & & 5 \\
\hline Río Claro - San Fernando & 1816 & Juan José Vergara & 16 & 9 & 8 & & & & & & 23 \\
\hline San Fernando & 1819 & Manuel Ruz & & 1 & & & & & & & 1 \\
\hline Río Claro - San Fernando & 1820 & Tiburcio Catalán & & 22 & & & & & & & 22 \\
\hline La Serena & 1820 & Francisca Navarrete & 1 & & & & & & & & 1 \\
\hline La Serena & 1820 & Margarita Muñoz & 1 & & & & & & & & 1 \\
\hline Placilla - San Ferrnando & 1821 & José Ruz & 1 & 45 & & & & & & & 46 \\
\hline Placilla - San Fernando & 1821 & Ignacio Arenas & & & 4 & 9 & & & & & 13 \\
\hline San Fernando & 1821 & Miguel Gálvez & 17 & 12 & & & 34 & & & & 63 \\
\hline Río Claro - San Fernando & 1822 & Tiburcio Catalán & & 22 & & & & & & & 22 \\
\hline San Felipe & 1825 & Juan Rodríguez Brito & & & & & & & & 1 & 1 \\
\hline Paniagua - San Fernando & 1825 & Ma Tránsito Cervantes & & & 2 & 3 & & & & & 5 \\
\hline San Felipe & 1826 & Isidro Osorio & & 18 & 1 & & & & & & 19 \\
\hline San Felipe & 1827 & Ma Antonia Muñoz & 4 & & & 3 & & & 1 & & 8 \\
\hline Coquimbo & 1827 & Eusebia Nieto & 1 & & & & & & & & 1 \\
\hline Encón - San Felipe & 1827 & Bernarda Ximénez & & 12 & 8 & & & & & & 20 \\
\hline San Felipe & 1828 & Martín de Oliva & & 16 & 16 & & & & & & 32 \\
\hline San Felipe & 1829 & José Carmona & & 1 & & & & & & & 1 \\
\hline La Serena & 1828 & Agustín Tirado & 1 & & & & & & & & 1 \\
\hline La Serena & 1831 & Javiera Martínez & 1 & & 3 & & & & & & 4 \\
\hline San Felipe & 1831 & Manuel José Núñez & & 3 & 3 & & & & & & 6 \\
\hline San Felipe & 1832 & Andrés Traslaviña & & 2 & & 1 & & & & & 3 \\
\hline Santiago & 1832 & Carmen Alarcón & & & & 1 & & & & & 1 \\
\hline La Serena & 1832 & Dionisio Argandoña & & 1 & & & & & & & 1 \\
\hline San Felipe & 1833 & Nicolasa Nieto & & 15 & 15 & & & & 1 & & 31 \\
\hline Santiago & 1833 & Carmen Alarcón & & & & 1 & & & & & 1 \\
\hline Curimón - San Felipe & 1835 & Joaquín Herrera & & & & & & & & 3 & 3 \\
\hline La Serena & 1836 & Francisco Bascuñán y $\mathrm{O}$ & 1 & & & & & & & & 1 \\
\hline Mendoza & 1836 & Felipe Segura & 1 & & & & & & & & 1 \\
\hline La Serena & 1836 & Mateo Vallejo & 2 & & & & & & & & 2 \\
\hline Aconcagua - San Felipe & 1838 & Constanza Muñoz & & 2 & & 1 & & & & & 3 \\
\hline San Felipe & 1838 & José Mansilla & & 9 & 5 & & & & & & 14 \\
\hline Aconcagua - San Felipe & 1838 & Juan Muñoz & & 7 & & & & & & & 7 \\
\hline Diaguitas - La Serena & 1841 & Andrés Callejas & 1 & & & & & & & & 1 \\
\hline Santiago & 1844 & Calixto Erazo & & 4 & 3 & 2 & & & & & 9 \\
\hline Mendoza & 1845 & José Godoy & 2 & & & & & & & & 2 \\
\hline Mendoza & 1848 & Francisca Pelliza & & & & 1 & & & & & 1 \\
\hline Santiago & 1848 & Quinta de Asolas & & & & 11 & & & & & 11 \\
\hline Mendoza & 1853 & José Manuel Funes & 1 & & & & 2 & & & & 3 \\
\hline Mendoza & 1854 & Manuel Silvestre Videla & 1 & & & & & & & & 1 \\
\hline Total cantidad & & & 165 & 349 & 150 & 41 & 36 & 7 & 7 & 9 & 764 \\
\hline Total \% & & & 21,6 & 45,6 & 19,6 & 5,3 & 4,7 & 0,9 & 0,9 & 1,1 & 100 \\
\hline
\end{tabular}

Fuentes: Fondos Notariales y Judiciales (AN, AHM, AGPSJ y APJSJ). Referencias: BER: Peral Bergamoto; CER: Peral Cerezo; RED: Peral Redondo; ARM: Peral Armenio; POR: Peral Portugués; CU: Peral Cuaresmero; CHI: Peral Chino. Otros: Peral de Guarda: Suasnabar, 1798 (1); Herrera, 1835 (3); Peral Invernizo: Ojeda, 1791 (3); Peral a libra: Ramírez, 1811 (1); Peral Joaquino: Rodríguez Brito, 1828 (1). 
Cuadro 2. Variedades de manzanos en Chile (1712-1843)

\begin{tabular}{|c|c|c|c|c|c|c|c|c|c|c|}
\hline Localidad & Año & Fruticultor & DUL & ACI & JOA & GUA & CAM & PER & QUI & Total \\
\hline Quillota & 1712 & Cecilia Covarrubias Lisperguer & 28 & 28 & & & & & & 56 \\
\hline Curimón & 1791 & Jerónima Ojeda & 5 & & 11 & 6 & & & & 22 \\
\hline Río Claro - S. Fdo. & 1798 & Nicolás Suasnabar & & & & 1 & & & & 1 \\
\hline Cucha Cucha - Itata & 1824 & Ramón Freire & & & & & & 60 & & 60 \\
\hline San Felipe & 1831 & Manuel José Núñez & 5 & & & & & & & 5 \\
\hline San Felipe & 1832 & Andrés Traslaviña & 6 & & & & & & & 6 \\
\hline La Serena & 1833 & Bernardo Solar & & & & & & 1 & & 1 \\
\hline Valle Aconcagua & 1836 & José Rosario García & & 1 & & & & & & 1 \\
\hline Santiago & 1837 & Mercedes Coo & & & & & 5 & & & 5 \\
\hline San Felipe & 1843 & Carmen Galdame & 11 & & & 4 & & & & 15 \\
\hline Santiago & 1848 & Quinta de Asolas & & & & & & & 1 & 1 \\
\hline La Hermita - Stgo. & 1848 & José Ignacio Eyzaguirre & 77 & 4 & & & & & & 81 \\
\hline Total cantidad & & & 132 & 33 & 11 & 11 & 5 & 61 & 1 & 254 \\
\hline Total \% & & & 51,9 & 12,9 & 4,3 & 4,3 & 1,9 & 24,0 & 0,4 & 100 \\
\hline
\end{tabular}

Fuentes: Fondos Notariales y Judiciales (AN, AHM, AGPSJ y APJSJ).

Referencias: DUL: Manzano Dulce; ACI: Manzano Acido; JOA: Manzano Joaquina; GU: Manzano de Guarda; CAM: Manzano Camueso; PER: Manzano Pero; QUI: Manzano Quillotano.

Cuadro 3. Variedades de membrillos en Chile y Cuyo (1793-1844)

\begin{tabular}{llllccc}
\hline Jurisdicción & Localidad & Fruticultor & Año & Variedad & Cantidad & Precio \\
\hline San Fernando & Villa S Fdo & María Josefa Celis & 1793 & Lúcumo & 1 & \\
San Felipe & Curimón & Francisco Borja Fuentecilla & 1800 & Lúcumo & 10 & $10 \times$ R 4 \\
Santiago & La Hermita & Antonio Aranguiz & 1838 & Dulce & 1 & s/d \\
San Juan & San Juan & Dionisio Sarmiento & 1844 & Lúcumo & 2 & $2 \times 4$ R \\
\hline
\end{tabular}

Fuentes: Fondos Notariales y Judiciales (AN, AGPSJ y APJSJ).

actualmente de valor económico significativo, pese a su gran potencial (maqui, frutilla blanca). Así ha ocurrido ya en vitivinicultura, con la revaloración del torrontés, promovido a la categoría de cepa emblemática de los vinos blancos de Argentina. En el campo de la fruticultura todavía no se ha alcanzado un resultado equivalente, pero ya han comenzado a verse indicios. Entre ellos, cabe mencionar a un municipio de Bolivia que viene de declarar el 2011 como "año de la manzana camuesa".

Agradecimientos. Este trabajo fue financiado por el proyecto FONDECYT 1080210. Mario Solar, Katherine Quinteros, Cristian Chávez y Natalia Soto son ayudantes de dicho proyecto.

\section{Literatura Citada}

Agustin, M.

1722 Libro de los secretos de la agricultura, casa de campo y pastoril (1617). Barcelona, Imprenta Juan Piferrer.

Agüero, C. et al.

2003 "Identity and Parentage of Torrontés Cultivars in Argentina", American Journal of Enology and Viticulture, American Society for Enology and Viticulture, 54: 318321.
Alonso de Herrera, G.

1818 Agricultura general. Corregida según el texto original de la primera edición publicada en 1513 por el mismo autor y adicionada por la Real Sociedad Económica Matritense. Madrid, Imprenta Real.

Cabello Sáenz de Santa María, F.

2011 "Las variedades de vid cultivadas en la península ibérica a través de los libros de agricultura y ampelografía". I 
Conferencia Internacional "Patrimonio Cultural de la Vid y el Vino", Almendralejo, Badajoz, 8 al 11 de febrero 2011.

Carabaza Bravo, J. M.; García Sánchez, E.; Hernández, E.; Jiménez Ramírez, A.

2004 Árboles y arbustos de Al Ándalus. Madrid, CSIC.

Ibáñez, J.; De Andres, M.T.; Zinelabidine; J.A.; Cabezas, L.; Gafario, L.; Muñoz, G.; Cabello, F.; Martinez Zapater, J.M.

2011 "Estudio de parentesco de variedades de vid mediante marcadores de ADN". I Conferencia Internacional
“Patrimonio Cultural de la Vid y el Vino”, Almendralejo, Badajoz, 8 al 11 de febrero 2011.

Lacoste, P.; Yuri, J. A.; Aranda, M.; Castro, A.; Quinteros, K.; Solar, M.; Soto, N.; Gaete, J.; Rivas, J.

2010 "Variedades de uva en Chile y Argentina (1550-1850): genealogía del torrontés". Mundo Agrario 20: 1. ISSN 15155994.

\section{Notas}

1 El peral joaquino puede tener relación con el peral joaquino portugués, variedad originaria de Carvalhais (Mirandela, Portugal) http://www.jb.utad.pt/pt/db/fruteiras_res2.asp?ID=1

2 La unidad de valor monetario más usual en el Reino de Chile era el peso de ocho reales.
3 Tasación de bienes de don Bernardo Ramírez, Guacargüe, villa de San Fernando, partido de Colchagua, 20 de junio de 1811. AN, FNSFdo, Legajo 53, Pieza 1, Folios 11, 11v, 18 y $18 \mathrm{v}$. 
